\title{
Two-Year Caries Clinical Study of the Efficacy of Novel Dentifrices Containing 1.5\% Arginine, an Insoluble Calcium Compound and 1,450 ppm Fluoride
}

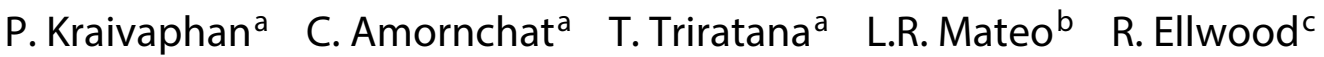 \\ D. Cummins ${ }^{c}$ W. DeVizio ${ }^{c} \quad$ Y.-P. Zhang ${ }^{c}$ \\ ${ }^{a}$ Faculty of Dentistry, Mahidol University, Bangkok, Thailand; ${ }^{b}$ LRM Statistical Consulting, Hoboken, N.J., and \\ ${ }^{c}$ Colgate-Palmolive Technology Center, Piscataway, N.J., USA
}

\section{Key Words}

Arginine $\cdot$ Caries · Fluoride · Prevention · Treatment

\begin{abstract}
A 2-year double-blind randomized three-treatment controlled parallel-group clinical study compared the anti-caries efficacy of two dentifrices containing $1.5 \%$ arginine, an insoluble calcium compound (di-calcium phosphate or calcium carbonate) and 1,450 ppm fluoride (F), as sodium monofluorophosphate, to a control dentifrice containing 1,450 ppm F, as sodium fluoride, in a silica base. The 6,000 participants were from Bangkok, Thailand and aged $6-12$ years initially. They were instructed to brush twice daily, in the morning and evening, with their randomly assigned dentifrice. Three trained and calibrated dentists examined the children at baseline and after 1 and 2 years using the National Institute of Dental Research Diagnostic Procedures and Criteria. The number of decayed, missing and filled teeth (DMFT) and surfaces (DMFS) for the three study groups were very similar at baseline, with no statistically significant differences among groups. After 1 year, there were no statistically significant differences in caries increments among the three groups. After 2 years, the two groups using the dentifrices containing $1.5 \%$ arginine, an insoluble calcium compound
\end{abstract}

and 1,450 ppm $F$ had statistically significantly $(p<0.02)$ lower DMFT increments (21.0 and $17.7 \%$ reductions, respectively) and DMFS increments (16.5 and 16.5\%) compared to the control dentifrice. The differences between the two groups using the new dentifrices were not statistically significant. The results of this pivotal clinical study support the conclusion that dentifrices containing $1.5 \%$ arginine, an insoluble calcium compound and $1,450 \mathrm{ppm} F$ provide significantly greater protection against caries lesion cavitation, in a low to moderate caries risk population, than dentifrices containing 1,450 ppm F alone.

Copyright ๑ 2013 S. Karger AG, Basel

The introduction of fluoride $(\mathrm{F})$ as a caries-preventive agent is one of the great public health success stories. Fluoride toothpastes, in particular, are believed by experts to have contributed significantly to the dramatic decline in dental caries observed around the world [Bratthall et al., 1996]. However, a recent survey conducted in the United States has confirmed a previous finding that dental caries is still the most common chronic disease in children

Clinical trial registration No. TCTR20130801001.

\begin{tabular}{ll}
\hline KARGER & $\begin{array}{l}\text { ○ 2013 S. Karger AG, Basel } \\
0008-6568 / 13 / 0476-0582 \$ 38.00 / 0 \quad \text { Karger }\end{array}$ \\
E-Mail karger@karger.com & $\begin{array}{l}\text { This is an Open Access article licensed under the terms of the } \\
\text { Creative Commons Attribution-NonCommercial 3.0 Un- } \\
\text { ported license (CC BY-NC) (www.karger.com/OA-license), } \\
\text { applicable to the online version of the article only. Distribu- } \\
\text { tion permitted for non-commercial purposes only. }\end{array}$
\end{tabular}


[United States Department of Health and Human Services, 2000, 2010], and several epidemiological studies conducted over the past decade indicate that dental caries remains a significant challenge. Bagramian et al. [2009] found increases in caries for children and adults, quoting numerous studies to support this view. The data point to gross disparities in prevalence and treatment of dental caries globally. A recent review [Cummins, 2013] identified diverse and changing global patterns of caries development, driven by the opposing forces of increased sugar consumption and effective oral hygiene and the use of fluoride, as well as divergent trends in caries experience, driven by deeper-rooted social and environmental factors. This increase in dental caries has signaled concerns of a pending public health crisis and the need to invigorate previously successful public health programs [Bagramian et al., 2009]. Whilst such measures can undoubtedly be successful again, new strategies are needed to supplement existing measures to reduce caries risk and improve dental health globally [National Institute of Health Consensus Development Panel, 2001; Pitts and Stamm, 2004]. More effective oral hygiene products, especially dentifrices that deliver superior caries prevention, have an important role to play in continued progress with the caries problem [Whelton, 2004].

Fluoride targets the tooth surface to arrest and reverse the caries process. However, fluoride has limitations under pathogenic conditions, because it does not target dental plaque as its primary mode of action. New strategies to deliver superior caries prevention would ideally complement the effects of fluoride while targeting plaque pathogenicity. Despite sustained research in this area, few conceptual approaches to reduce plaque pathogenicity have progressed to clinically validated cost-effective products [Cummins, 2013]. Recently, however, a new dentifrice technology based upon $1.5 \%$ arginine and an insoluble calcium compound to reduce plaque pathogenicity and enhance re-mineralization, in combination with fluoride, has been developed and clinically validated. This clinical validation comprises a series of five studies of 6 months duration which assessed the efficacy in arresting and reversing (re-mineralizing) early, reversible enamel lesions and root caries lesions. The studies demonstrated that innovative dentifrices containing $1.5 \%$ arginine, an insoluble calcium compound and fluoride [Cummins, 2013] provide superior caries benefits in children and adults to dentifrices containing fluoride alone [Hu et al., 2013; Souza et al., 2013; Srisilapanan et al., 2013; Yin et al., 2013a, b]. Further, it includes in situ clinical studies which showed that these dentifrices inhibit de-mineralization and enhance re-mineralization more effectively than dentifrices with fluoride alone [Cantore et al., 2013]. In these clinical efficacy studies, two sources of insoluble calcium compound, di-calcium phosphate and calcium carbonate, were evaluated and shown to be interchangeable without impact on efficacy.

The aim of this pivotal 2-year double-blind randomized three-treatment controlled parallel-group clinical study was to investigate the efficacy of two dentifrices containing $1.5 \%$ arginine, an insoluble calcium compound and $1,450 \mathrm{ppm} \mathrm{F}$ in preventing caries lesion cavitation compared to a dentifrice with $1,450 \mathrm{ppm} F$ alone. The two sources of insoluble calcium compound, di-calcium phosphate and calcium carbonate, which were previously evaluated, were directly compared in this study.

\section{Subjects and Methods}

This double-blind randomized three-treatment controlled parallel-group clinical study was conducted in Bangkok, Thailand, where the fluoride level in tap water is $<0.3 \mathrm{ppm}$ [Metropolitan Waterworks Authority, 2007]. Prior to initiation of the study, the protocol and pertinent documents were reviewed and approved by the Mahidol University Institutional Review Board.

Schools were selected to take part in the study based on the known caries risk of their students, levels of cooperation of the staff, students and parents/guardians, and low population mobility determined during previous studies in the area and pilot investigations. Study participants volunteering to take part were schoolchildren aged 6-12 years, with recruitment focused on classes with 8- and 9-year-old children predominantly. Written informed consent to take part was obtained from a parent or legal guardian. The children were selected based on the following inclusion and exclusion criteria.

\section{Inclusion Criteria}

(1) Children in elementary or middle school who had at least four permanent molars fully erupted and at least one permanent central and/or lateral incisor erupted. (2) Informed consent form signed by parent or legal guardian to permit participation of their child in the study.

\section{Exclusion Criteria}

(1) Presence of orthodontic appliances. (2) Any condition which, in the opinion of the investigator, would hinder participation by the potential subject. The reason for any such exclusion was to be carefully documented by the investigator. (3) Participation in any other clinical study during the 3 months preceding the clinical study. (4) History of allergies or other adverse reactions to arginine, oral care products or their ingredients.

Qualified subjects were randomly assigned to one of the three study treatments using a randomization table generated centrally by the study statistician. Two groups received one of the two dentifrices each containing $1.5 \%$ arginine, an insoluble calcium 
compound - either di-calcium phosphate (test dentifrice 1; Colgate-Palmolive, New York, N.Y., USA) or calcium carbonate (test dentifrice 2; Colgate-Palmolive) - and 1,450 ppm F as sodium monofluorophosphate. The third group received a silicabased dentifrice containing 1,450 ppm F as sodium fluoride (fluoride control dentifrice; Procter \& Gamble, Cincinnati, Ohio, USA).

Each subject was provided with three $150-\mathrm{ml}$ tubes of their assigned dentifrice and a manual toothbrush (Colgate soft-bristled adolescent-size toothbrush) every 2 months for their exclusive use at home. The tubes of dentifrice were completely wrapped in white tape so that neither the examiners nor the subjects were aware of the identity of the product they were using. Participants were instructed to brush their teeth twice daily, in the morning and evening, at home. No instructions on rinsing behavior were provided and subjects brushed as per their established daily routine. School nurses monitored the tooth brushing of subjects by interview, collection of used toothpaste tubes and assessment of oral hygiene. Classroom teachers also reinforced the importance of at home brushing to the participants and parents/guardians to ensure that, within the constraints of a home use unsupervised setting, subjects brushed their teeth twice daily. When the subjects received new supplies of dentifrice and toothbrushes, the clinical team checked and reviewed returned/used dentifrices to help ensure compliance.

The sample size per group was estimated, based on pilot data and previous investigations conducted in the population, using the following assumptions: (1) a 2-year study period, (2) three treatment groups, (3) coefficient of variation $=1.1,(4)$ power $=$ 0.8 , (5) $\alpha=0.05$, two sided t test, (6) a $10 \%$ difference between products, (7) $10 \%$ annual attrition of subjects. With these assumptions, the required minimum sample size to satisfy the American Dental Association Council on Dental Therapeutics criterion for superior efficacy (10\% difference between test and control products) at the end of the 2-year period of product use was approximately 1,920 subjects per group.

Clinical examinations were performed in school classrooms, using portable dental surgery equipment, at baseline and after 1 and 2 years of product use using the criteria and methods described by the National Institute of Dental Research [1991].

\section{Clinical Examination and Scoring Procedure for Coronal \\ Caries}

Caries examinations were conducted by three trained and calibrated examiners in accordance with the established criteria [United States Department of Health, Education and Welfare, 1978; National Institute of Dental Research, 1991] after cleaning and drying the teeth. Each of the three examiners performed clinical examinations for the same subjects (approximately 2,000) at baseline and after 1 and 2 years of product use. The examinations were conducted using a halogen light source with an attached dental mouth mirror (Miltex Inc., York, Pa., USA) and a \#23 singleend dental explorer (Miltex Inc.). A visual-tactile assessment of dental caries status was made using the explorer to confirm continuity of the enamel surface. The status of all tooth surfaces for each permanent tooth was assessed, with the exception of third molars. Subsequently decayed, missing and filled teeth (DMFT) and surfaces (DMFS) scores were calculated for each participant and then the mean DMFT and DMFS scores were calculated for each dentifrice group.

\section{Examiner Calibration}

Training and calibration of the three caries examiners was conducted by an experienced clinician (Dr. Augusto Elias Boneta, University of Puerto Rico, Puerto Rico). The first part of the training and calibration was a discussion of diagnostic criteria, examination methods and procedures. In the second part, the examiners were calibrated for inter- and intra-examiner reliability using a representative sample of subjects. The inter- and intra-examiner reliability was quantified using an unweighted kappa statistic. A threshold of $\geq 0.75$ was used to qualify the examiners.

\section{Oral Soft and Hard Tissue Assessment}

The three dental examiners visually examined the oral cavity and peri-oral area of the subjects, whom they had evaluated for caries, using the same dental light and dental mirror used for the caries assessments. This examination included an evaluation of the soft and hard palate, gingival mucosa, buccal mucosa, mucogingival fold areas, tongue, sublingual and submandibular areas, salivary glands, tonsillar and pharyngeal areas. In the event that the clinical examiner identified oral conditions that required further investigation or treatment, children were referred to their family dentist or community dental service.

\section{Adverse Events}

Adverse events were obtained from interviews with each subject and from a dental examination by each examiner at each appointment.

\section{Statistical Analysis}

Statistical analyses were performed for both the DMFT and DMFS scores for all subjects completing the study. Comparisons of the treatment groups with respect to baseline scores were performed using conventional analysis of variance (ANOVA). The results were later confirmed using non-parametric tests (KruskalWallis). Comparisons between the treatment groups with respect to gender were performed using $\chi^{2}$ tests and for age using ANOVA. Comparisons among treatment groups with respect to baselineadjusted incremental DMFT and incremental DMFS scores at the 1- and 2-year examinations were performed using analysis of covariance (ANCOVA). Post-ANCOVA pairwise comparisons of the study treatments were performed using the Tukey test for multiple comparisons. Non-parametric tests were also run to confirm the pairwise comparisons with probability corrections for multiple comparisons [Gibbons, 1997]. All statistical tests of hypotheses were two-sided and employed a level of significance of $\alpha=0.05$.

\section{Results}

Nine elementary schools in Bangkok, Thailand with a total of 9,082 subjects were first approached to take part; 8,989 consented and 6,090 met the inclusion criteria. Of these, 6,000 were assigned to one of the three study groups, whereas 90 who were unavailable for examination were excluded from the study (fig. 1).

Inter- and intra-examiner calibration was performed for each of the three examination periods. The kappa sta- 


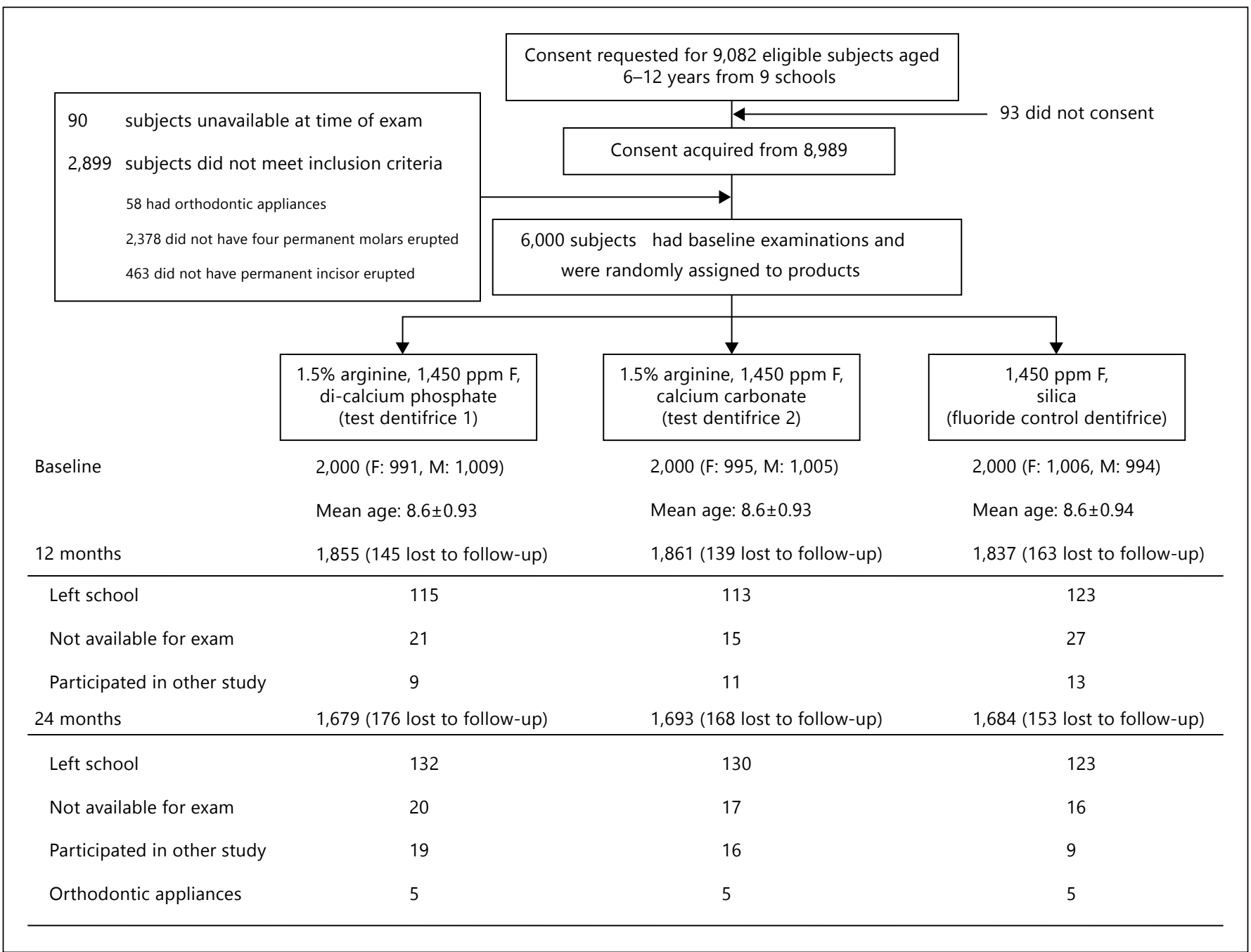

Fig. 1. Disposition of subjects in the 2-year clinical study.

tistics for inter- and intra-examiner reproducibility of DMFT and DMFS were all $>0.83$, indicating a high level of agreement within and between the three examiners.

Throughout the study, no adverse effects on the oral hard or soft tissues were observed or reported.

\section{Baseline Data}

The gender and age of the study subjects who completed the study are presented in figure 1 for the three study groups. The three groups did not differ significantly with respect to these characteristics. The percentages of children aged $6,7,8,9,10,11$ and 12 years at baseline were 0.59 , $10.01,32.94,38.60,16.87,0.89$ and $0.10 \%$, respectively.

Summaries of the baseline data for the DMFT and DMFS scores are presented in table 1 . The mean baseline
DMFT scores for test dentifrice 1, test dentifrice 2 and the fluoride control dentifrice were $0.51 \pm 0.97,0.49 \pm 0.94$ and $0.49 \pm 0.97$, respectively. The mean baseline DMFS scores were $0.73 \pm 1.62$ for test dentifrice $1,0.67 \pm 1.56$ for test dentifrice 2 and $0.71 \pm 1.63$ for the fluoride control dentifrice. No statistically significant differences in either DMFT or DMFS were indicated among the three groups (table 2).

\section{One-Year Data Incremental DMFT and DMFS Scores}

The mean incremental DMFT scores measured after 1 year of product use are shown in table 1 and were $0.25 \pm$ $0.72,0.26 \pm 0.69$ and $0.24 \pm 0.63$ for test dentifrice 1 , test dentifrice 2 and the fluoride control dentifrice, respectively. After only 1 year of product use, no statistically 
Table 1. Summary of the baseline DMFT and DMFS scores and 1- and 2-year caries increments for subjects who completed the 2-year clinical study

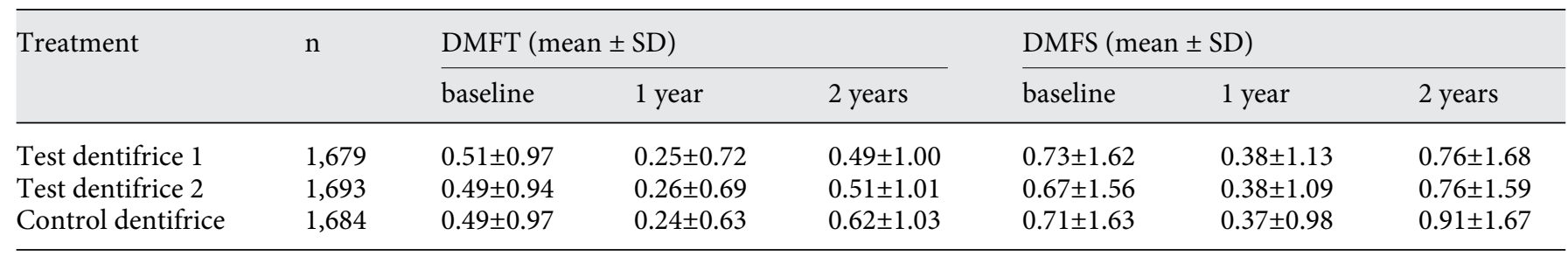

Table 2. Summary of the statistical significances of differences between subjects in the three groups for those who completed the 2 -year clinical study

\begin{tabular}{|c|c|c|c|c|}
\hline Comparison between dentifrices & \multicolumn{4}{|c|}{ Statistical significance of the pairwise comparisons } \\
\hline Test dentifrice $1 \times$ test dentifrice 2 & 0.99 & 0.82 & 0.94 & 0.96 \\
\hline Test dentifrice $1 \times$ control dentifrice & 0.91 & 0.001 & 0.99 & 0.009 \\
\hline Test dentifrice $2 \times$ control dentifrice & 0.85 & 0.007 & 0.886 & 0.02 \\
\hline
\end{tabular}

significant differences were indicated among the three dentifrice groups with respect to reduction in incremental DMFT scores.

The mean incremental DMFS scores measured after 1 year of product use are shown in table 1 and were $0.38 \pm$ $1.13,0.38 \pm 1.09$ and $0.37 \pm 0.98$ for test dentifrice 1 , test dentifrice 2 and the fluoride control dentifrice, respectively. Likewise, no statistically significant differences were indicated among the three dentifrice groups with respect to reduction in incremental DMFS scores after 1 year of product use (table 2).

\section{Two-Year Data Incremental DMFT and DMFS Scores}

The mean incremental DMFT scores measured after 2 years of product use are presented in table 1 . They were $0.49 \pm 1.00,0.51 \pm 1.01$ and $0.62 \pm 1.03$ for test dentifrice 1 , test dentifrice 2 and the fluoride control dentifrice, respectively. Relative to the fluoride control dentifrice, both test dentifrice 1 and test dentifrice 2 exhibited statistically significant reductions (table 2) in incremental DMFT scores (21.0 and $17.7 \%$, respectively). However, no statistically significant difference was observed between the two test dentifrices in incremental DMFT scores after 2 years of product use (table 2 ). The statistical significance of differences was subsequently confirmed using nonparametric methods [Gibbons, 1997] with comparisons between both test dentifrices and the fluoride control dentifrice of $\mathrm{p}<0.001$. No significant difference between the two test dentifrices $(p>0.05)$ was observed.

The mean incremental DMFS scores measured after 2 years of product use are also shown in table 1 . They were $0.76 \pm 1.68,0.76 \pm 1.59$ and $0.91 \pm 1.67$ for test dentifrice 1 , test dentifrice 2 and the fluoride control dentifrice, respectively. Relative to the fluoride control dentifrice, both test dentifrice 1 and test dentifrice 2 exhibited statistically significant reductions (table 2) in incremental DMFS scores after 2 years of product use ( 16.5 and $16.5 \%$, respectively). The incremental DMFS scores for the two test dentifrices after 2 years of product use, however, were not statistically significantly different (table 2). The statistical significance of differences was again subsequently confirmed using non-parametric methods [Gibbons, 1997] with comparisons between both test dentifrices and the fluoride control dentifrice of $p<0.001$. No significant difference between the two test dentifrices $(p>0.05)$ was observed.

\section{Discussion}

Fluoride has long been recognized for its anti-caries benefits and the efficacy of fluoride dentifrices in preventing caries has been well documented [Marinho et al., 
2009; Walsh et al., 2010]. However, despite active research on new ingredients and innovative formulations, significant enhancements to the clinical efficacy of dentifrices formulated with $1,450 \mathrm{ppm} \mathrm{F}$, the maximum permitted level of fluoride in mass-marketed dentifrices, have not, until now, been forthcoming. A new technology based upon $1.5 \%$ arginine and an insoluble calcium compound in combination with 1,450 ppm F has demonstrated great promise in clinical studies of 6 months duration, providing statistically significant and clinically meaningful anti-caries efficacy compared to dentifrices with 1,450 ppm F alone [Cummins, 2013].

The results of this pivotal 2-year clinical study demonstrate (1) that the two dentifrices containing $1.5 \%$ arginine, an insoluble calcium compound and $1,450 \mathrm{ppm} \mathrm{F}$ provide statistically significant $(\mathrm{p}<0.05)$ reductions in incremental DMFT and DMFS scores compared to the control dentifrice containing 1,450 ppm F alone and (2) that the two arginine-containing dentifrices with either di-calcium phosphate or calcium carbonate provided similar levels of efficacy in preventing the formation of new cavitated caries lesions. It was important to evaluate efficacy for both sources of insoluble calcium compound, di-calcium phosphate and calcium carbonate, in this pivotal study as both abrasive systems will be available to consumers. Importantly, the percent reductions in mean caries increment for the two dentifrices relative to the fluoride-only control, over the relatively short time period for cavitated caries lesion formation of 2 years, exceed the accepted criterion for superior anticaries efficacy of 10\% [American Dental Association Council on Dental Therapeutics, 1988; Proskin et al., 1995], highlighting the clinical relevance of the results. The results are also highly significant relative to the effects of 1,450 ppm F toothpaste which provides statistically significant reductions of approximately $25 \%$ relative to fluoride-free toothpaste [Marinho et al., 2009], typically over time periods longer than 2 years, an effect recognized by caries experts to be the primary factor in reducing caries on a population basis [Bratthall et al., 1996].

Although there is strong scientific evidence that the anti-caries efficacy of marketed toothpastes with sodium fluoride, typically with a silica base, is comparable to the anti-caries efficacy of marketed toothpastes with sodium monofluorophosphate, typically with a calcium base [Saporito et al., 2000; Marinho et al., 2009], there are some authors who have suggested the superiority of these sodium fluoride formulations [Stookey et al., 1993]. For this reason, a silica-based sodium fluoride dentifrice was used as the control formulation in this study. Amongst the five clinical studies of 6 months duration and the in situ clinical studies which demonstrated the great promise of this technology, several have used calcium-based sodium monofluorophosphate toothpastes as matched controls and have also demonstrated superior anti-caries efficacy for the toothpaste containing 1.5\% arginine [Cantore et al., 2013; Souza et al., 2013; Srisilapanan et al., 2013].

The results of this pivotal 2-year caries clinical study which assessed the development of cavitated caries lesions are highly consistent with the outcomes of the shorter-term clinical studies that focused on the re- and de-mineralization of early, reversible enamel and dentin lesions. Importantly, this is the first time that a new caries prevention dentifrice containing $1,450 \mathrm{ppm} F$ has been shown to provide superior efficacy at multiple stages within the caries continuum versus a dentifrice with $1,450 \mathrm{ppm} F$ alone. Specifically, this clinical study has shown superior efficacy of dentifrices containing $1.5 \%$ arginine, an insoluble calcium compound and $1,450 \mathrm{ppm} \mathrm{F}$ compared to $1,450 \mathrm{ppm} \mathrm{F}$ alone, over the 2-year time frame appropriate to the irreversible stage of cavitation, and has also demonstrated both qualitatively and quantitatively comparable results to the five clinical studies over the 6-month time frame appropriate to the arrest and reversal of the early stages of the caries process.

Three studies, using quantitative light-induced fluorescence, have each shown that the new dentifrice containing $1.5 \%$ arginine, an insoluble calcium compound and $1,450 \mathrm{ppm} F$ is significantly more effective in arresting and reversing coronal caries lesions in children than a dentifrice containing 1,450 ppm $\mathrm{F}$ alone [Srisilapanan et al., 2013; Yin et al., 2013a, b]. In one study, after 6 months of product use, improvements from baseline in $\Delta \mathrm{Q}$ (lesion volume) were $50.7,32.3$ and $11.4 \%$ for the new arginine-containing dentifrice, a matched positive control dentifrice and a matched negative control dentifrice, respectively. The differences between the new dentifrice and the positive control were statistically significant $(p=0.003)$ [Yin et al., 2013a]. In a second study, after 6 months of product use, improvements from baseline in $\Delta \mathrm{Q}$ (lesion volume) were $50.6,34.0$ and $13.1 \%$ for the new arginine-containing dentifrice, a silica/sodium fluoride positive control dentifrice and a matched negative control dentifrice, respectively. The differences between the new dentifrice and the positive control were statistically significant ( $\mathrm{p}=0.008$ ) [Yin et al., 2013b]. In a third study, after 6 months of product use, improvements from baseline in $\Delta \mathrm{Q}$ (lesion volume) were 44.6 and 
28.9\% for the new arginine-containing dentifrice and a matched positive control dentifrice, respectively. The difference between the new dentifrice and the positive control was statistically significant $(\mathrm{p}<0.001)$ [Srisilapanan et al., 2013].

Two root caries studies in adults have each shown that the new dentifrice containing $1.5 \%$ arginine and $1,450 \mathrm{ppm} F$ in a calcium base is significantly more effective in arresting and reversing root caries lesions than a dentifrice containing 1,450 ppm F alone [Hu et al., 2013; Souza et al., 2013]. In one study, after 6 months of product use, clinical hardness measures showed that only one lesion $(0.7 \%)$ was worse in the new dentifrice group compared to 9.0 and $18.2 \%$ in a silica/sodium fluoride positive and a matched negative control group, respectively. In addition, $61.7,56.0$ and $27.0 \%$ of lesions showed improvement for the new arginine-containing dentifrice, the positive control dentifrice and the negative control dentifrice, respectively. The differences in the distribution of lesion change scores between the new dentifrice and the positive control were statistically significant $(\mathrm{p}=$ 0.006) [Hu et al., 2013]. In the second study, after 6 months of product use, $70.5 \%$ of root caries lesions improved for subjects using the new dentifrice compared to $58.1 \%$ for subjects in a matched positive control group. The difference in the number of root caries lesions being hardened in the new dentifrice and positive control groups was statistically significant $(\mathrm{p}<0.05)$ [Souza et al., 2013].

The fact that the three enamel caries studies and the two root caries studies described above demonstrated statistically significant differences between the argininecontaining dentifrices and positive control dentifrices in only 6 months is consistent with the time scale of formation and reversal of early non-cavitated caries lesions. It was not expected that, in the present study, differences between products in respect to cavitation would be seen until the 2-year time point. This is consistent with other conventional caries clinical trials [Marinho et al., 2009] and reflects the time required for cavitated lesions to develop to clinically detectable thresholds using conventional visual-tactile methods. The 6-month enamel and root caries clinical trial methods that assess re- and demineralization of early, reversible caries lesions show great potential to significantly reduce development cycles and provide a greater understanding of modes of action of anti-caries products [Ellwood et al., 2012].

In this 2-year clinical study, the DMFT increments of between 0.49 and 0.62 are relatively small. This likely reflects that the population participating in this study comprised subjects with low to moderate caries risk, rather than with high risk seen in some populations around the world. Interestingly, the caries scores in this study population are representative of Thailand as a whole, as a recent survey [Bureau of Dental Health, Ministry of Public Health, 2012] showed that 12-year-olds have mean DMFT scores of 1.3 and 1.4 in Thailand and in Bangkok, respectively.

In fact, it is unusual to be able to demonstrate superior efficacy of anti-caries products relative to controls in such a low to moderate caries risk population, and most studies are conducted in high caries risk groups [Marinho et al., 2009] to increase discrimination between products [Ellwood et al., 2012]. The ability of this study to demonstrate superior efficacy for the arginine-containing dentifrice relative to the fluoride only control could, thus, be viewed as a strength of the study. Notwithstanding this, as described previously, the efficacy of the arginine-containing dentifrice was demonstrated in three quantitative light-induced fluorescence studies in populations with higher caries risk and also in two populations with root caries. The consistent findings of clinical studies in such diverse populations suggest that the findings of these studies would be applicable to a wide range of populations and age groups.

The results of this pivotal 2-year clinical study, as well as the results of the five 6-month clinical studies, are also consistent with the results of in situ clinical studies which demonstrated that these arginine-containing dentifrices inhibit de-mineralization and enhance re-mineralization more effectively than dentifrices with fluoride alone [Cantore et al., 2013]. The superior caries protection benefits of these dentifrices are delivered through a combination of the well-known effects of fluoride and a unique mechanism of action of the arginine and insoluble calcium compound [Cummins, 2013].

Independent clinical studies provide further insights into this unique mechanism of action. Firstly, in situ ammonia production from salivary arginine via the arginine deiminase system in dental plaque is positively associated with reduced caries experience [Nascimento et al., 2009; Gordan et al., 2010], indicating the importance of ammonia production in caries prevention. Further, it has been shown that fluoride-free toothpaste containing $1.5 \%$ arginine significantly increased arginine deiminase system activity in caries-active individuals, indicating that exogenous arginine can reduce caries risk by increasing arginine deiminase system activity [Nascimento et al., 2009]. Additionally, a 2-year clinical study has demonstrated that this fluoride-free toothpaste containing $1.5 \%$ arginine provides equivalent anti-caries efficacy to a silica- 
based toothpaste containing 1,100 ppm [Acevedo et al., 2005], indicating that exogenous arginine may provide clinically meaningful caries prevention.

\section{Conclusion}

The results of this pivotal 2-year caries clinical study support the conclusion that dentifrices containing $1.5 \%$ arginine, an insoluble calcium compound and $1,450 \mathrm{ppm} \mathrm{F}$ as sodium monofluorophosphate provide significantly greater protection against caries lesion cavitation, in a low to moderate caries risk population than dentifrices containing 1,450 ppm F alone.

\section{Acknowledgement}

The authors would like to thank both the nine schools involved in this study and the clinical trial team of Mahidol University for their support and enthusiasm in conducting this study.
They also would like to thank Dr. Augusto Elias Boneta for his dedication and expertise in training and calibrating the clinical examiners.

\section{Author Contributions}

Dr. Petcharat Kraivaphan: leading examiner; Dr. Choltivha Amornchat: examiner and logistics; Dr. Terdphong Triratana: principal investigator and examiner; Mr. Luis R. Mateo: statistics; Dr. Roger Ellwood: manuscript, discussion, data interpretation and statistics; Dr. Diane Cummins: manuscript, discussion, data interpretation and statistics; Dr. William DeVizio: sponsor and discussion in study design; Dr. Yun-Po Zhang: study design, monitoring, manuscript, discussion, data interpretation and corresponding author.

\section{Disclosure Statement}

This research was funded by Colgate-Palmolive. Drs. Kraivaphan, Amornchat, Triratana and Boneta and Mr. Mateo were funded by Colgate-Palmolive to conduct this study. Drs. Ellwood, Cummins, DeVizio and Zhang are employees of Colgate-Palmolive.

\section{References}

Acevedo AM, Machedo C, Rivera LE, Wolff M, Kleinberg I: The inhibitory effect of an arginine bicarbonate/calcium carbonate CaviStat-containing dentifrice on the development of dental caries in Venezuelan school children. J Clin Dent 2005;16:63-70.

-American Dental Association Council on Dental Therapeutics: Report of workshop aimed at defining guidelines for caries clinical trials: superiority and equivalency claims for anticaries dentifrices. J Am Dent Assoc 1988;117: 663-665.

- Bagramian R, Garcia-Godoy F, Volpe AR: The global increase in dental caries. A pending public health crisis. Am J Dent 2009;21: 3-7.

Bratthall D, Hänsel-Petersson G, Sundberg H: Reasons for the caries decline: what do the experts believe? Eur J Oral Sci 1996;104:416422.

Bureau of Dental Health, Ministry of Public Health: The 7th Thailand National Oral Health Survey, Thailand, 2012. http://dental. anamai.moph.go.th/oralhealth/PR/E-book/ Survey/survey7th.pdf.

Cantore R, Petrou I, Lavender S, Santarpia P, Liu Z, Vandeven M, Cummins D, Sullivan R, Utgikar N: In situ clinical effects of new dentifrices containing $1.5 \%$ arginine and 1,450 p.p.m. fluoride on enamel de- and remineralization and plaque metabolism. J Clin Dent 2013, accepted for publication.

Cummins D: Dental caries: a disease which remains a public health concern in the 21 st century - the exploration of a breakthrough tech- nology for caries prevention. J Clin Dent 2013, accepted for publication.

Ellwood RP, Gomez J, Pretty IA: Caries clinical trial methods for the assessment of oral care products in the 21 st century. Adv Dent Res 2012;24:32-35.

Gibbons JD: Nonparametric Methods for Quantitative Analysis, ed 3. New York, American Sciences Press, 1997, pp 196-208.

Gordan VV, Garvan CW, Ottenga ME, Schulte R, Harris PA, McEdward D, Magnusson I: Could alkali production be considered an approach for caries control? Caries Res 2010;44: 547-554.

Hu DY, Yin W, Li X, Feng Y, Zhang YP, Cummins D, Mateo LR, Ellwood RP: A clinical investigation of the efficacy of a dentifrice containing $1.5 \%$ arginine and 1,450 p.p.m. fluoride, as sodium monofluorophosphate in a calcium base, on primary root caries. J Clin Dent 2013, accepted for publication.

Marinho VC, Higgins JP, Sheiham A, Logan S: Fluoride toothpastes for preventing dental caries in children and adolescents. Cochrane Database Syst Rev 2009;1:CD002278.

Metropolitan Waterworks Authority: Fluoride content of tap water in Bangkok. Bangkok, Metropolitan Waterworks Authority, 2007.

Nascimento MM, Gordan VV, Garvan CW, Browngardt CM, Burne RA: Correlations of oral bacterial arginine and urea catabolism with caries experience. Oral Microbiol Immunol 2009;24:89-95.

National Institute of Dental Research: Oral health surveys of the National Institute of Dental Re- search, diagnostic criteria and procedures. Publication No. 91-2870, 1991.

National Institute of Health Consensus Development Panel: National Institute of Health Consensus Development Conference statement. Diagnosis and management of dental caries throughout life, March 26-28, 2001. J Am Dent Assoc 2001;132:1153-1161.

Pitts NB, Stamm JW: International Consensus Workshop on Caries Clinical Trials (ICWCCT) - final consensus statements: agreeing where the evidence leads. J Dent Res 2004; 83(special issue C):125-128.

Proskin HM, Kingman A, Naleway C, Wozniak $\mathrm{W}$ : Comparative attribute for the description of the relative efficacy of therapeutic agents: general concepts and definitions, and application to the American Dental Association guidelines for the comparison of the clinical anticaries efficacy of fluoride dentifrices. J Clin Dent 1995;6:176-184.

Saporito R, Boneta ARE, Feldman CA, Cinotti W, Sintes J, Stewart B, Volpe AR, Proskin HM: Comparative anticaries efficacy of sodium fluoride and sodium monofluorophosphate dentifrices. A two-year caries clinical trial on children in New Jersey and Puerto Rico. Am J Dent 2000;13:221-226.

Souza MLR, Cury JA, Tenuta LMA, Zhang YP, Mateo LR, Cummins D, Ellwood RP: Comparing the efficacy of a dentifrice containing $1.5 \%$ arginine and 1,450 p.p.m. fluoride to a dentifrice containing 1,450 p.p.m. fluoride alone in the management of primary root caries. J Dent 2013, accepted for publication. 
Srisilapanan P, Korwanich N, Yin W, Chuensuwonkul C, Mateo, LR, Zhang YP, Cummins D, Ellwood RP: Comparison of the efficacy of a dentifrice containing $1.5 \%$ arginine and 1,450 p.p.m. fluoride to a dentifrice containing 1,450 p.p.m. fluoride alone in the management of early coronal caries as assessed using quantitative light-induced fluorescence. J Dent 2013, accepted for publication.

Stookey GK, DePaola PF, Featherstone JD, Fejerskov O, Möller IJ, Rotberg S, Stephen KW, Wefel JS: A critical review of the relative anticaries efficacy of sodium fluoride and sodium monofluorophosphate dentifrices. Caries Res 1993;27:337-360.

United States Department of Health and Human Services: Oral health in America: a report of the Surgeon General. Washington, DC, 2000.
United States Department of Health and Human Services: Promoting and enhancing the oral health of the public: HHS Oral Health Initiative. Washington, DC, 2010

United States Department of Health, Education and Welfare, Public Health Service, Food and Drug Administration: Guidelines for the Clinical Evaluation of Drugs to Prevent Dental Caries. Rockville, 1978, vol 79, p 3075.

Walsh T, Worthington HV, Glenny AM, Appelbe P, Marinho VC, Shi X: Fluoride toothpastes of different concentrations for preventing dental caries in children and adolescents. Cochrane Database Syst Rev 2010; 1:CD007868.

Whelton H: Overview of the impact of changing global patterns of dental caries experience on caries clinical trials. J Dent Res 2004;83(special issue):29-34.
Yin W, Hu DY, Fan X, Feng Y, Zhang YP, Cummins D, Mateo LR, Pretty IA, Ellwood RP: A clinical investigation using quantitative lightinduced fluorescence (QLF) of the anticaries efficacy of a dentifrice containing $1.5 \%$ arginine and 1,450 p.p.m. fluoride as sodium monofluorophosphate. J Clin Dent 2013a, accepted for publication.

Yin W, Hu DY, Li X, Fan X, Zhang YP, Pretty IA, Mateo LR, Cummins D, Ellwood RP: The anti-caries efficacy of a dentifrice containing $1.5 \%$ arginine and 1,450 p.p.m. fluoride as sodium monofluorophosphate assessed using quantitative light-induced fluorescence (QLF). J Clin Dent 2013b; accepted for publication. 